\title{
The direct correlation functions and bridge functions for hard spheres near a large hard sphere
}

\author{
Douglas Henderson \\ Departamento de Física, Universidad Autónoma Metropolitana/Iztapalapa, Apartado Postal 55-534, \\ 09340 México DF, México
}

Kwong-yu Chan

Department of Chemistry, University of Hong Kong, Pokfulam Road, Hong Kong

Léo Degrève

Faculdade de Filosofia, Ciências e Letras de Ribeirão Preto, Universidade de São Paulo, 14040-901

Ribeirão Preto (SP), Brazil

(Received 22 March 1994; accepted 23 May 1994)

\begin{abstract}
The recent Monte Carlo data of Degrève and Henderson for the density profiles of hard spheres near a large hard sphere are used to obtain direct correlation functions and bridge functions for this system both directly, using the Ornstein-Zernike relation and an approximation, due to Verlet, for the bridge function.
\end{abstract}

\section{INTRODUCTION}

The calculation of the direct correlation function from a pair correlation function or a profile requires a knowledge of the bridge function which is defined below. Explicit formulas for the bridge function are available only for the coefficients in an expansion of this function. Only the low order coefficients are known. These coefficients are difficult to calculate and the convergence of the series may not be rapid. As a result it is common to use simple approximations. The one employed here, due to Verlet, seems to be the best simple approximation.

Density profiles, $\rho(x)$, for a hard sphere fluid near a large hard sphere have been reported in two recent studies. Attard ${ }^{\prime}$ has obtained $\rho(x)$ using integral equation techniques. Degrève and Henderson ${ }^{2}$ have obtained $\rho(x)$ using the Monte Carlo method. These studies give $\rho(x)$ for a wide range of densities of the bulk hard spheres, $\rho=\rho(\infty)$, and for a full range of the ratio, $R$, of the diameters of the large and the small hard spheres. The direct correlation function for the hard spheres near a hard wall was not reported in these studies. It seems to us to be worthwhile to report results for this function and for the closely related bridge function. This we do in this paper. The results reported here are valid only in the limit of small concentration of large spheres.

\section{METHOD}

We consider a single solute hard sphere in a solvent of hard spheres. Let $R$ be the ratio of the diameter of the solute hard sphere to that of the solvent hard sphere. By writing our basic formulas for variable $R$, we avoid the need to repeat formulas for the cases of solvent-solvent and solvent-solute pairs and achieve an economy. First we write the general expressions and do not specify any subscripts. If $R=1$, the correlation functions of the pair are those of the solvent and are not subscripted. If $R>1$, the correlation functions of the pair are those of solvent-solute pair and are subscripted with the symbol $w$.

Outside the hard sphere cores, $r \geqslant(R+1 / 2)$, the cavity function, $y(r)$, is equal to $g(r)=\rho(r) / \rho$. We obtain approximate values for $y(r)$ inside the core using

$$
\begin{gathered}
\ln y(r)=D, \quad 0 \leqslant r \leqslant \lambda, \\
\ln y(r)=A \frac{(r-\lambda)^{4}}{r}+B \frac{(r-\lambda)^{3}}{r}+C \frac{(r-\lambda)^{2}}{r}+D, \\
\lambda \leqslant r \leqslant \frac{R+1}{2},
\end{gathered}
$$

where $\lambda=(R-1 / 2)$ and the diameter of the small hard spheres has been taken, without loss of generality, to be $\sigma=1$. The distance $r$ is the separation of the centers of the two spheres. The parameter $D$ is given by

$$
D=\frac{8 \eta-9 \eta^{2}+3 \eta^{3}}{(1-\eta)^{3}}
$$

where $\eta=\pi \rho \sigma^{3} / 6$, which gives a very accurate value ${ }^{3,4}$ for $y(0)$. The parameter $C$ was chosen to be

$$
C=-\frac{3 \eta(2-\eta)}{(1-\eta)^{3}}\left(1+\frac{1+2 \eta}{1+\eta / 2} \lambda\right)
$$

This expression gives a very accurate value for $y^{\prime}(0)$ for $R=1{ }^{3,4}$ The $R$ dependence for $C$ was chosen in analogy to the corresponding Percus-Yevick (PY) expression. ${ }^{5}$ For $R>1$, Eq. (1a) gives $y^{\prime}(0)=0$ for $r \leqslant \lambda$ which is known to be correct. ${ }^{4}$ The remaining two parameters, $A$ and $B$, were chosen so that $y(r)$ and its slope at $(R+1 / 2)$ are continuous.

If $R=1$, Eq. (1) is the the same as the expression used by Grundke and Henderson (GH) (Ref. 4) which was found to be accurate by means of a computer simulation by Torrie and Patey. ${ }^{6}$ We expect Eq. (1) to be accurate for $R>1$ also. Labik et $a l^{7}$ have developed an alternative formula for $y(r)$.

To obtain the direct correlation functions, $c(r)$, we need an approximation to the bridge function. We use an approximation, due to Verlet, ${ }^{8}$ which was brought to our attention by a paper of Lee, ${ }^{9}$

$$
B(r)=-\frac{\gamma^{2}(r)}{2[1+\alpha \gamma(r)]},
$$


where $B(r)$ is the bridge function and differs from the parameter $B$ of Eq. (1). The bridge function is defined by

$$
\begin{aligned}
& \gamma(r)=h(r)-c(r)=\ln y(r)-B(r), \\
& h(r)= \begin{cases}-1, & r \leqslant(R+1) / 2 \\
y(r)-1, & r \geqslant(R+1) / 2,\end{cases}
\end{aligned}
$$

and $\alpha$ is a parameter which is usually taken to be $4 / 5$. This approximation has been used recently by Labik et al. ${ }^{10}$ The function $B(r)$ is defined in terms of $\chi(r)$. Thus, we must solve Eqs. (4)-(6) to obtain $\chi(r)$. The result is

$$
\gamma(r)=\frac{2 b(r)+\left[b^{2}(r)+8 c_{1} \ln y(r)\right]^{1 / 2}}{c_{1}},
$$

where

$$
b(r)=\alpha \ln y(r)-1
$$

and

$$
c_{1}=2 \alpha-1 \text {. }
$$

Once $\chi(r)$ is known, $B(r)$ can be calculated from Eq. (4) and $c(r)$, the direct correlation function, can be obtained from

$$
c(r)=\left\{\begin{array}{l}
-1-\gamma(r), \quad r \leqslant(R+1) / 2 \\
y(r)-1-\gamma(r), \quad r \geqslant(R+1) / 2,
\end{array}\right.
$$

For $R=1, c(r)$ can be obtained from the OrnsteinZernike (OZ) relation,

$$
h\left(r_{12}\right)=c\left(r_{12}\right)+\rho \int h\left(r_{13}\right) c\left(r_{23}\right) d \mathbf{r}_{3},
$$

since $y(r)$, and hence $h(r)$, is known from simulation studies. In principle, $c(r)$ can be obtained from $h(r)$ by taking the Fourier transform of Eq. (11) and solving the resulting algebraic equation,

$$
\tilde{c}(k)=\frac{\tilde{h}(k)}{1+\rho \tilde{h}(k)},
$$

where $\tilde{h}(k)$ and $\tilde{c}(k)$ are the Fourier transforms of $h(r)$ and $c(r)$, respectively, and then taking the inverse Fourier transform. In practice, some care is needed since, at high densities, the denominator in Eq. (12) is nearly zero for $k \approx 0$. As a result, small errors in $\tilde{h}(0)$, primarily due to truncation, produce large errors in $\tilde{c}(0)$ if simulation data are used. It is better to obtain $\tilde{h}(k)$ and $c(r)$ using some fit of the simulation data. For example, the Verlet-Weis (VW) (Ref. 11) fit of $h(r)$, which is constrained to give correct values for $\tilde{h}(0)$, can be used, as was done by GH.

Rather than use a constant value of $\alpha$, we choose $\alpha$ so that the value of $c(0)$, computed using Eqs. (4) $-(9)$ for $R=1$, matches that obtained by the procedure of $\mathrm{GH}$ or that of Groot $e t$ al. ${ }^{12}$ At high densities, $\alpha$ is close to $4 / 5$; however, $\alpha$ becomes increasingly large as the density decreases. The resulting values of $c(r)$ are virtually the same as those of GH and Groot et al.

For $R>1$, values for $c_{w}(r)$ and $B_{w}(r)$ could be obtained from the $\mathrm{OZ}$ relation, which is, in Fourier space,

$$
\tilde{c}_{w}(k)=\frac{\tilde{h}_{w}(k)}{1+\rho \tilde{h}(k)},
$$

where, the subscript $w$ is used for large-small pairs $(R>1)$ and no subscript is used for the $R=1$ (bulk or small hard spheres) functions. In principle, $h_{w}(r)$ obtained by simulation methods can be used to compute $\tilde{h}_{w}(k)$ and the VerletWeis fit can be used to obtain $\tilde{h}(k)$ for the denominator of Eq. (13).

The above procedure was the one we intended to use at the commencement of this work. In general, we were successful. Our calculated $c_{w}(r)$ and $B_{w}(r)$ were constant and equal to $c(0)$ and $B(0)$, respectively, for $r \leqslant \lambda$. We expected this because of the results of $\mathrm{GH}$. These constant values are a reflection of the fact that the small sphere has disappeared inside the large sphere when $r \leqslant \lambda$ and nothing changes. However, the $c_{w}(r)$ which we obtained in this manner was negative for $r \geqslant(R+1) / 2$. Although not inconceivable, such a behavior is unexpected and unusual. These values of $c_{w}(r)$ in the neighborhood of the contact value, $(R+1) / 2$, are small and are likely to be in error because of numerical problems. The large negative values for $0 \leqslant r \leqslant(R+1) / 2$ are dominant in determining $c_{w}(r)$.

One could obtain a VW fit of $h_{w}(r)$ and then determine $c_{w}(r)$. This was done by GH. However, their criterion for determining $h_{w}(R+1 / 2)$ is now known to be in error for $R$ large and at least for small concentrations of the large sphere. Their formulas and computer program would have to be modified. This we have not done yet.

However, the fact that $\gamma_{w}(r), c_{w}(r)$, and $B_{w}(r)$ for $0 \leqslant r \leqslant \lambda$ are constant and equal to $\chi(0), c(0)$, and $B(0)$, respectively, means that, at least for this range of $r$, Eqs. (4)(10) are valid with the same value of $\alpha$ as for the small hard spheres. It seems plausible that these equations are equally valid for $\gamma_{w}(r), c_{w}(r)$, and $B_{w}(r)$ for all values of $r$. This is the approximation used in this paper.

\section{RESULTS}

First $\alpha$ was calculated. The resulting values can be obtained from

$$
\alpha=\frac{17}{120 \eta}+0.5150-0.2210 \eta
$$

The first coefficient is exact; the other two are not. Values for the cavity function, the direct correlation function and bridge function are shown in Figs. 1-3. The results for these functions for $R=1$ are well known but are included for comparison and completeness. The values shown for $R=11.8$ were calculated using our simulation values for $y_{w}(r)$, extended for $0 \leqslant r \leqslant 6.4$ by means of Eq. (1), and $B_{w}(r)$ and $c_{w}(r)$ were then calculated using the Verlet approximation, Eq. (4). The functions $y_{w}(r), B_{w}(r)$, and $c_{w}(r)$ do not change for $r \leqslant \lambda$ because once the small sphere has "disappeared" inside the large sphere, nothing changes for $r \leqslant \lambda$.

If we obtain $c_{w}(r)$ directly from the simulation values of $h_{w}(r)$ using Eq. (13), we obtain curves which are similar to the results of Fig. 2. These values for $c_{w}(r)$ are constant and equal to $c(0)$ for $r \leqslant \lambda$ and then decrease for $0 \leqslant r-\lambda \leqslant 1$. The 


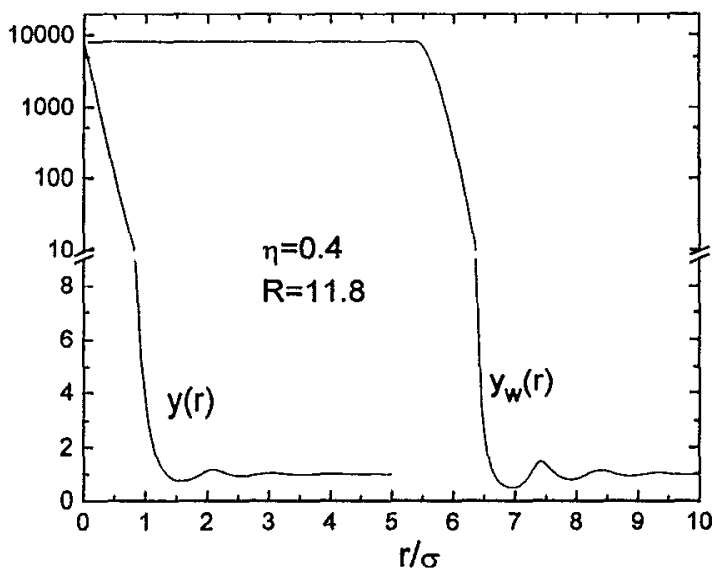

FIG. 1. Cavity functions for a fluid of hard spheres near a large hard sphere.

only difference from the results in Fig. 1 is that $c_{w}(r)$ at $r=(R+1) / 2$ is negative and small. This behavior, we presume is due to numerical problems and is not correct. Therefore, we prefer the values in Fig. 2.

\section{CONCLUSIONS}

The Verlet approximation seems useful for calculating $B(r)$ and $B_{w}(r)$ for hard sphere systems. Its utility for other potentials is less certain. The Verlet approximation with $\alpha$ constant is identical to the PY approximation at low densities and so gives an incorrect fourth virial coefficient. This is not too serious for hard spheres since the PY fourth virial coefficient is reasonably accurate. However, the PY fourth virial coefficient can have substantial errors for potentials with attractive regions. A state dependent $\alpha$, as was done here, may overcome this and other problems.

The question of whether the Verlet approximation is useful for calculating the bridge function, $B_{w w}(r)$, for a pair of large hard spheres in a fluid of hard spheres is open. In some ways, Eq. (4) is attractive. Attard et al. ${ }^{13}$ and Henderson ${ }^{14}$ have shown that $\gamma_{w w}(r)$ and $\ln y_{w w}(r)$ are proportional to $R$,

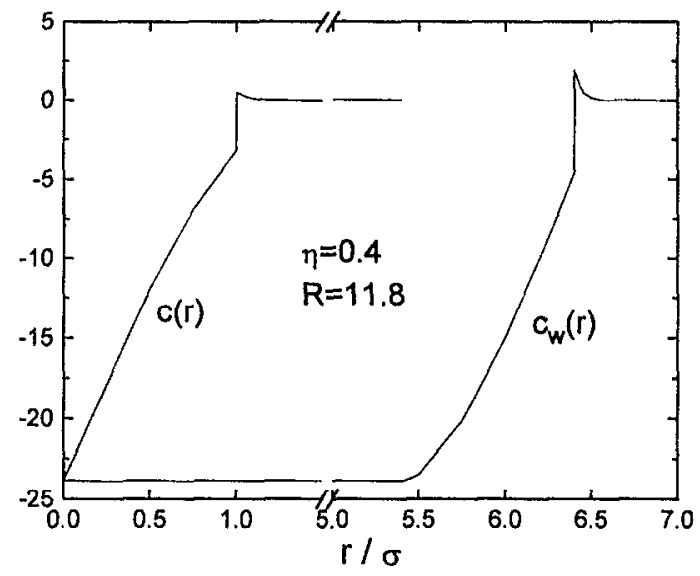

FIG. 2. Direct correlation functions for a fluid of hard spheres near a large hard sphere.

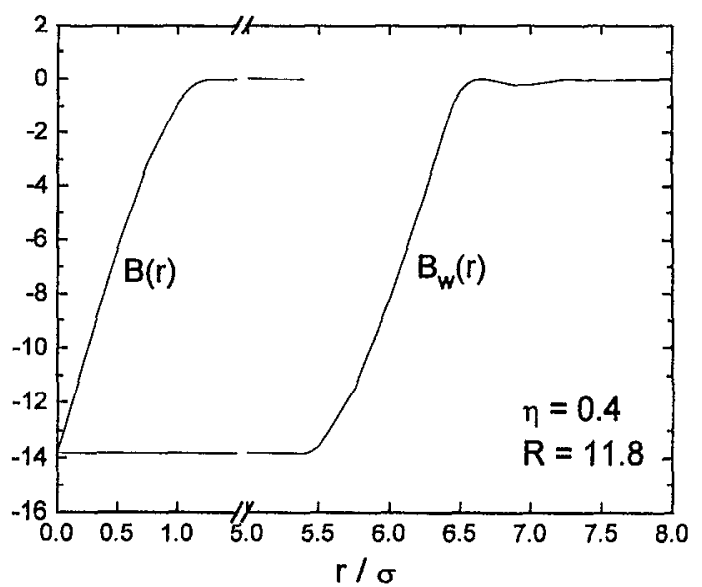

FIC. 3. Bridge functions for a fluid of hard spheres near a large hard sphere.

when $R$ is large. This means that $B_{w w}(r)$ must also be proportional to $R$, when $R$ is large. If $\alpha_{w w}$ is a constant and is of order $R$, Eq. (4) gives a $B_{w w}(r)$ which is of order $R$ at low densities (or possibly ambiguous depending on the limit of $\rho R^{2}$ and $\rho^{2} R^{2}$ as $\rho$ tends to zero and $R$ tends to infinity) and independent of $R$ at finite densities, which is incorrect. On the other hand, if $\alpha_{w w}$ is a constant, but not large, then Eq. (4) yields a $B_{w w}(r)$ which is proportional to $R^{2}$ at low densities (or possibly ambiguous depending on the limit of $\rho R$ as $\rho$ tends to zero and $R$ tends to infinity) and which is proportional to $R$ at finite densities, which is better but not completely satisfactory. If $\alpha_{w w}$ is not of order $R$ and proportional to $\rho^{-1}$ at low densities, which is the case for $B(r)$ and $B_{w}(r)$, then $B_{w w}(r)$ is proportional to $R$ at all densities, which seems attractive.

In fact, it was considerations such as these which lead us to an approximation, based on the Martynov-Sarkisov (MS) approximation, ${ }^{15}$

$$
B(r)=-\frac{[\ln y(r)]^{2}}{2[1+\alpha \ln y(r)]} .
$$

Without the second term in the denominator (i.e., the MS approximation), this approximation gives a $B_{w w}(r)$ which is proportional to $R^{2}$, which is incorrect. We abandoned Eq. (15) after we became aware of Eq. (4) as there is no point inventing new approximations when there are existing approximations which are likely to be as good. Our point is that thinking about $B_{w w}(r)$ can lead to Eq. (4) or similar approximations.

In summary, we feel that the Verlet approximation is useful for approximating hard sphere $B(r)$ and $B_{w}(r)$, especially if $\alpha$ is not kept constant, and that the same values of $\alpha$ may be used for both functions. The Verlet approximation may well be useful for $B_{w w}(r)$ also. However, it is unlikely that the same values of $\alpha$ will be appropriate for $B_{w w}(r)$. Intuitively, we feel that the hypernetted chain approximation, ${ }^{16}$

$$
B_{w w}(r)=0,
$$


may turn out to be the best approximation but have no quantitative arguments to support this conjecture.

\section{ACKNOWLEDGMENTS}

The support of CONACYT of México, the Committee on Research and Conference Grants of the University of Hong Kong, and the Conselho Nacional de Desenvolvimento Cientifico e Tecnologico of Brazil is acknowledged with thanks. D.H. is grateful for the hospitality of the Department of Chemistry of the University of Hong Kong during his stay in May 1993 when this work was commenced.

\footnotetext{
${ }^{1}$ P. Attard, J. Chem. Phys. 91, 3083 (1989).

${ }^{2}$ L. Degrève and D. Henderson, J. Chem. Phys. 100, 1606 (1994).

${ }^{3}$ E. Meeron and A. J. F. Siegert, J. Chem. Phys. 48, 3139 (1968).

${ }^{4}$ E. W. Grundke and D. Henderson, Mol. Phys. 24, 269 (1972).
}

5. Lebowitz, Phys. Rev. 133, A895 (1964).

${ }^{6}$ G. Torrie and G. N. Patey, Mol. Phys. 34, 1623 (1977).

${ }^{7}$ S. Labik, W. R. Smith, and R. J. Speedy, J. Chem. Phys. 88, 1944 (1988).

${ }^{8}$ L. Verlet, Mol. Phys. 41, 183 (1980).

${ }^{9}$ L. L. Lee, J. Chem. Phys. 97,8606 (1992).

${ }^{10}$ S. Labik, A. Malijevsky, and W. R. Smith, Mol. Phys. 73, 87, 495 (1991); R. Pospisil, A. Malijevsky, and S. Labik, and W. R. Smith, ibid. 74, 253 (1991); S. Labik, A. Malijevsky, R. Pospisil, and W. R. Smith, ibid. 74, 261 (1991).

${ }^{11}$ L. Verlet and J.-J. Weis, Phys. Rev. A 5, 939 (1972).

${ }^{12}$ R. D. Groot, J. P. van der Eerden, and N. M. Faber, J. Chem. Phys. 87, 2263 (1987).

${ }^{13}$ P. Attard, D. R. Bernard, C. P. Ursenbach, and G. N. Patey, Phys. Rev. A 44, 8224 (1991).

${ }^{14}$ D. Henderson, J. Chem. Phys. 97, 1266 (1992).

${ }^{15}$ G. A. Martynov and G. N. Sarkisov, Mol, Phys. 49, 1495 (1983).

${ }^{16}$ For a full set of references, see J. A. Barker and D. Henderson, Rev. Mod. Phys. 48, 587 (1976). 